\title{
Ab-Interno Trabeculotomy
}

\author{
Richard L. Rabin, Jaehong Han, and Douglas J. Rhee
}

\subsection{Introduction}

Multiple experimental and morphological studies of primate and human eyes have demonstrated that the anatomical location of the greatest resistance to aqueous outflow is at the juxtacanalicular trabecular meshwork $[1,2]$. To overcome this resistance, goniotomy was first introduced in 1936 as the first surgical procedure directed at the trabecular meshwork, with significant success in infants and young children with congenital glaucoma, but relatively poor outcomes in adults [3]. Opening Schlemm's canal to direct aqueous outflow can be accomplished in adults from either an ab-externo or an ab-interno approach. In 1989, Rosenquist et al. studied the aqueous outflow resistance of enucleated human eyes and showed that complete (12 clock hours) internal trabeculotomy reduced $71 \%$ of the aqueous outflow resistance in eyes with $25 \mathrm{mmHg}$ intraocular pressure. They also reported that with just one clock hour of trabeculotomy, $41 \%$ of the total effect was obtained [4].

Trabectome (NeoMedix Corporation, San Juan Capistrano, CA, USA) received approval by the US Food and Drug Administration in April 2004. The first published trial of US patients was in January 2006 for the surgical treatment of openangle glaucoma. The Trabectome removes a segment of the trabecular meshwork and the inner wall of the Schlemm's canal using an ab-interno approach, enhancing aqueous outflow via increased access to the Schlemm's canal and the collector channels (Fig. 4.1).

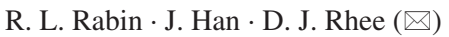

Department of Ophthalmology and Visual Sciences, University Hospitals, Cleveland Medical Center, Cleveland, OH, USA

Case Western Reserve University School of Medicine, Cleveland, OH, USA

e-mail: Douglas.Rhee@uhhospitals.org 


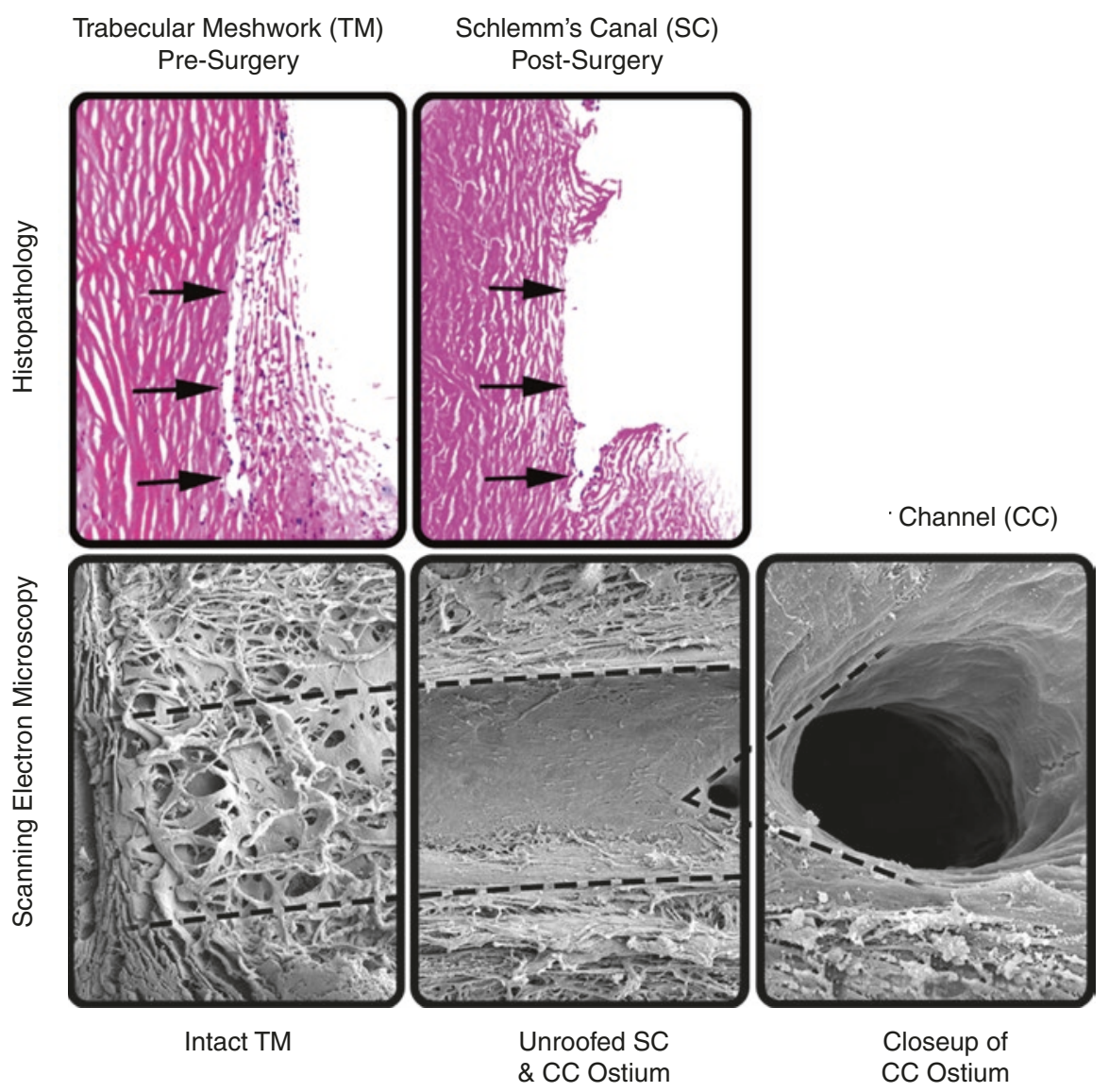

Fig. 4.1 Upper panels: Histopathology images of the trabecular meshwork and Schlemm's canal before and after Trabectome procedure. Lower panels: Electron microscopy images of the anterior chamber angle structures before and after Trabectome procedure. (Copyright NeoMedix Corporation, San Juan Capistrano, CA, USA; reproduced with permission)

\subsection{Trabectome Device}

The Trabectome device consists of a single-use, disposable handpiece that combines electrocautery, irrigation, and aspiration. The handpiece is connected to a generator with a frequency of $550 \mathrm{kHz}$ that allows adjustments in $0.1 \mathrm{~W}$ increments and is controlled via a three-stage foot pedal that initiates irrigation, aspiration, and electrocautery in sequence (Figs. 4.2 and 4.3). Irrigation and aspiration permit removal of debris and regulation of temperature. The tip of the Trabectome is angled at $90^{\circ}$ to create a protective triangular-shaped footplate for easier insertion into Schlemm's canal. The footplate is coated permitting smoother movement within the canal and protecting the outer wall of Schlemm's canal from the thermal energy. 


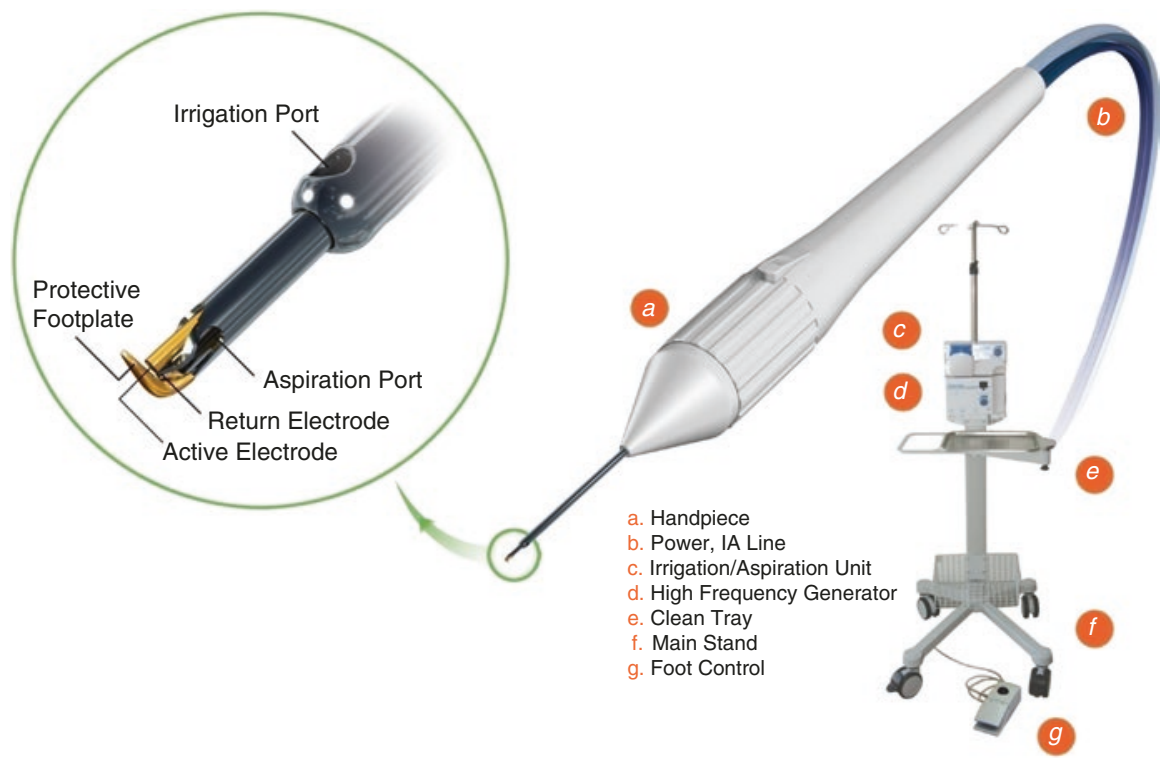

Fig. 4.2 Trabectome console and foot pedal. (Copyright NeoMedix Corporation, San Juan Capistrano, CA, USA; reproduced with permission)

The electrode tip of the Trabectome creates plasma that ablates the trabecular meshwork, thereby creating a less traumatic procedure [5]. Continuous infusion minimizes thermal injury to surrounding structures and aspiration removes tissue debris to allow for more consistent action of the electrode. In studies by Minckler and Francis [6, 7], Trabectome-treated specimens showed less damage to surrounding structures compared to eyes treated by goniotomy blade (Fig. 4.1).

\subsection{Trabectome Procedure}

Some surgeons pretreat patients with topical apraclonidine $0.5 \%$ or brimonidine $0.1 \%$ to reduce intraoperative reflux bleeding. Bleeding can also be reduced by adequate pressurization of the anterior chamber at the conclusion of the procedure. The patient's head is tilted $30-45^{\circ}$ away from the surgeon and the operating microscope is tilted $30-45^{\circ}$ toward the surgeon to create a near $90^{\circ}$ viewing angle to the viewing axis of the eye (Fig. $4.3 \mathrm{~b}, \mathrm{c}$ ).

When performing both Trabectome and phacoemulsification (phacoTrabectome), we prefer to perform the Trabectome portion first to optimize corneal clarity. A $1.8 \mathrm{~mm}$, two-step clear corneal incision is created using a keratome blade. The inner third of the incision can be flared to improve mobility of the handpiece and visualization of the surgery by eliminating corneal striae from torquing of the wound. The Trabectome irrigation is activated by depressing the black colored 
a

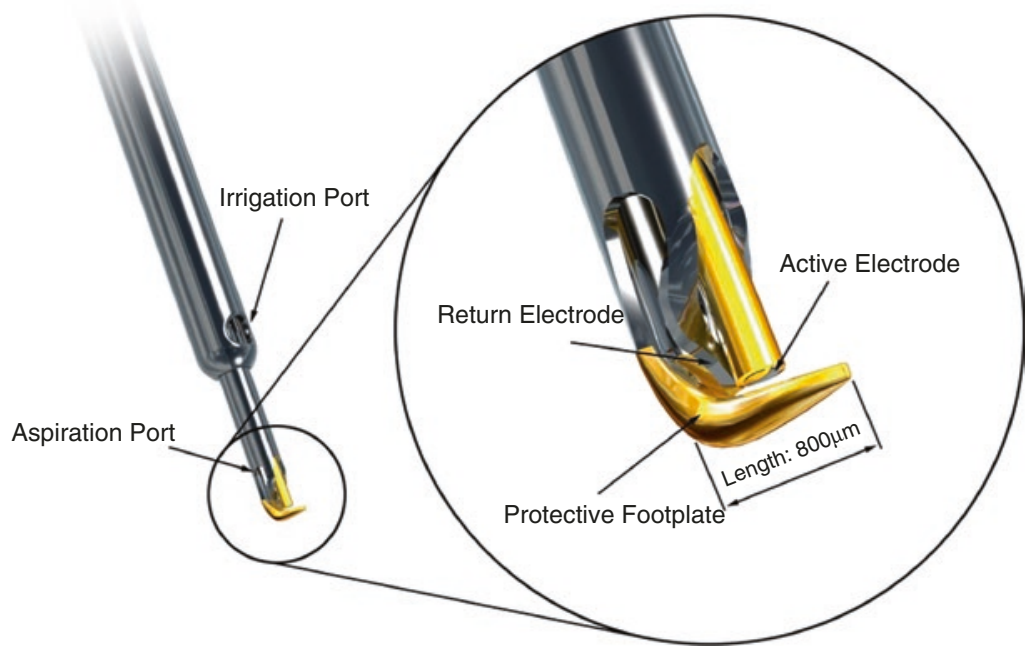

b

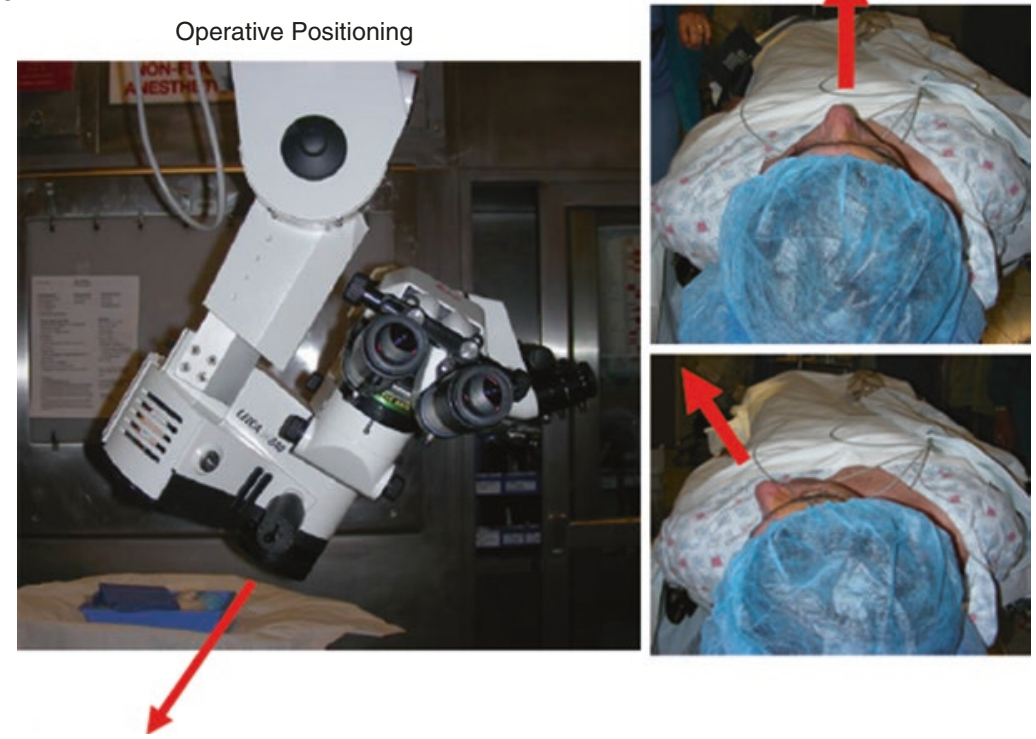

Fig. 4.3 (a) Trabectome handpiece distal end: Electrode, aspiration port, and irrigation port are visible. Footplate dimensions: heel to tip, $800 \mu \mathrm{m}$; footplate maximum width, $230 \mu \mathrm{m}$; footplate maximum thickness, $110 \mu \mathrm{m}$. Gap between electrocautery pole and footplate: $150 \mu \mathrm{m}$. (Copyright NeoMedix Corporation, San Juan Capistrano, CA, USA; reproduced with permission) (b) Microscope and head positioning: Operating room microscope is tilted between 35 and $45^{\circ}$ (left panel) while the patient's head is similarly turned $35-45^{\circ}$ away from the surgeon. In this example, the patient's position is shown in the typical faceup position for cataract surgery (right upper panel) and appropriate positioning for the right eye for the Trabectome procedure (right lower panel). (c) Intraoperative positioning of the operating room microscope and patient's head as shown in b. (d) Schematic of the centripetal and lateral vectors of motion of the probe while in Schlemm's canal in order to accommodate the circular shape of the canal 

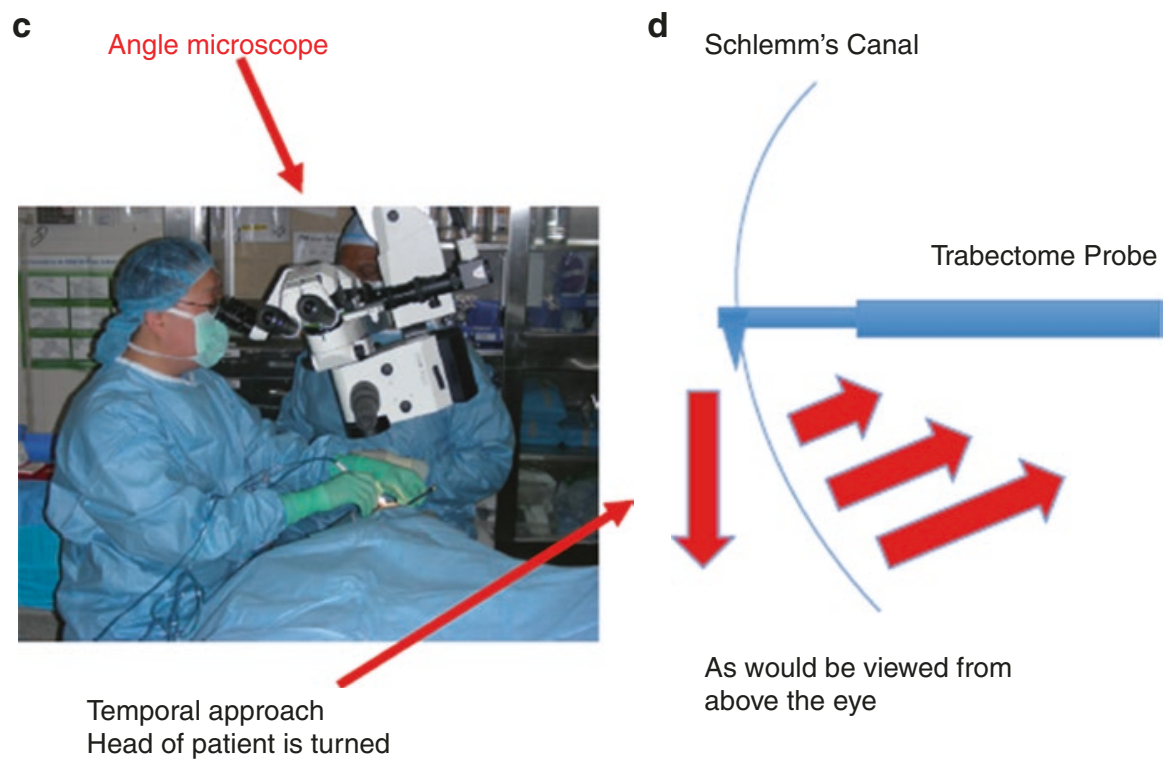

As would be viewed from above the eye

Fig. 4.3 (continued)

button on the foot pedal and the probe is inserted into the anterior chamber under direct visualization. Cohesive viscoelastic (Healon) may be used to deepen the nasal anterior chamber angle and as a coupling agent for the gonioscopy lens. The active irrigation of the Trabectome system helps to keep the anterior chamber formed during the procedure and is designed to reduce the need for viscoelastic in the anterior chamber.

After the probe's irrigation port enters the anterior chamber, the direct view surgical gonioscopy lens, e.g., Swan Jacob gonioprism, is applied on the cornea to view the nasal angle. After the trabecular meshwork is identified, the probe is inserted into Schlemm's canal and the foot pedal is depressed fully to activate aspiration and electrocautery. The foot pedal has three positions_- "off" in which neither aspiration nor electrocautery is active, "first position" in which aspiration is active, but not electrocautery, and "second position" in which both aspiration and electrocautery are activated. Torquing of the eye and failure to advance the tip with gentle pressure may indicate that the tip is lodged in the back wall of the Schlemm's canal. This can be corrected by backing the probe up toward the area of previously treated tissue and trying again. During the procedure, it is crucial to eliminate any outward push on Schlemm's canal as this can damage the wall of Schlemm's canal and the collector channel system. It is therefore important to apply a slight inward pull (i.e., toward the pupil) during ablation to offset any tendency to push or rub against the wall of Schlemm's canal (Fig. 4.3d).

After successfully completing one direction of treatment, the Trabectome probe can be rotated $180^{\circ}$ and advanced in the opposite direction. Following a successful 


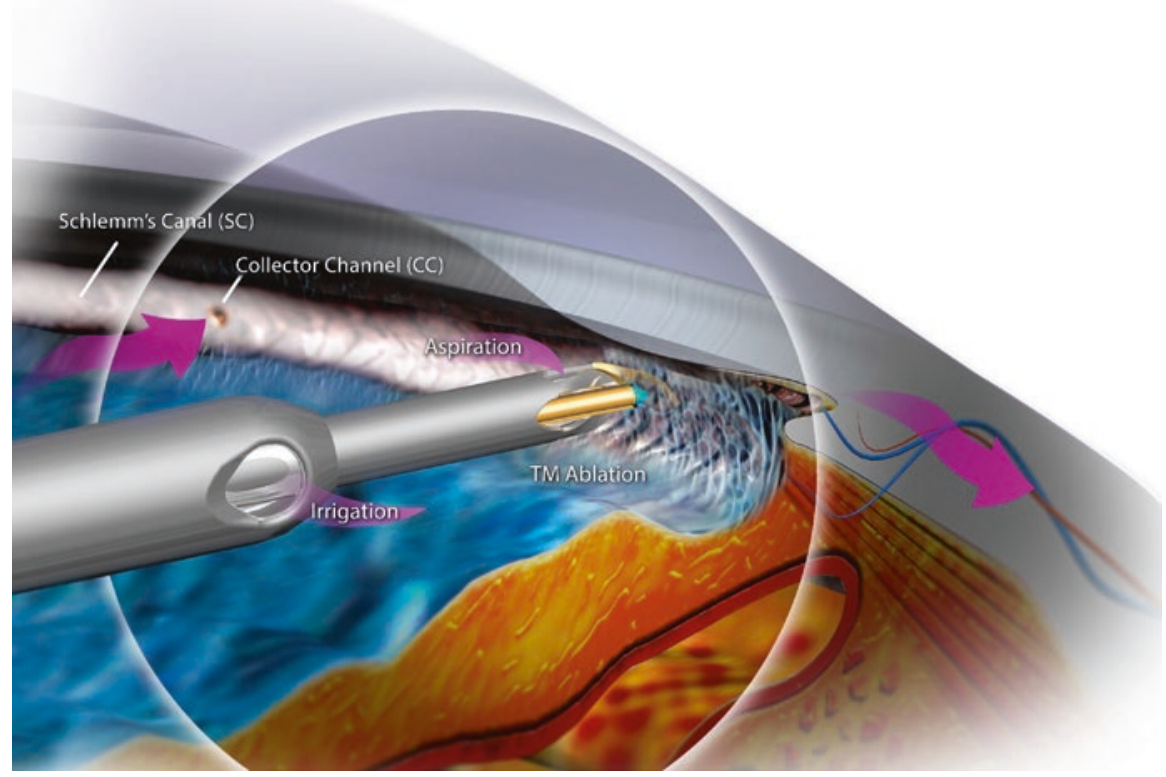

Fig. 4.4 Ablation of trabecular meshwork using the Trabectome probe. (Copyright NeoMedix Corporation, San Juan Capistrano, CA, USA; reproduced with permission)

procedure, the outer wall of Schlemm's canal will appear opalescent (Fig. 4.4). After the treatment, the probe is retracted so that the irrigation port is just inside the wound. The gonioscopy lens is then removed and the probe is removed from the eye under direct visualization. The microscope and patient's head can then be repositioned if cataract surgery is to be performed. A properly constructed wound should seal with stromal hydration.

Most surgeons experienced in Trabectome procedures report treating $60-120^{\circ}$ of angle. The length of the ablation arc has not been found to be significantly correlated with the subsequent intraocular pressure (IOP) decrease [8]. Up to $800 \mathrm{~mW}$ of electrocautery power can be used. Coagulation necrosis can result if the energy used exceeds $1000 \mathrm{~mW}$, which manifests as darkening along the edge of the ablated tissue.

To reduce intraocular bleeding, air-bubble tamponade in the anterior chamber can be considered or the eye can be pressurized to $20-25 \mathrm{mmHg}$, provided optic nerve/visual field damage is not advanced and other risk factors for complications associated with transiently elevated IOP are absent, e.g., post-cataract surgery anterior ischaemic optic neuropathy or retinal vascular occlusion following previous eye surgery. Postoperative care generally includes the use of topical antibiotic and steroid eye drops, with a tapering regimen according to the surgeon's preference. In general, we recommend a rapid taper of corticosteroids. Some surgeons advocate prescribing pilocarpine $1-2 \%$ postoperatively to prevent the formation of peripheral anterior synechiae. 


\subsection{Surgical Planning}

In patients with mild cataract and glaucoma, many options exist-phacoemulsification alone, minimally invasive glaucoma surgery (MIGS) alone, combined cataract surgery and MIGS, conventional glaucoma surgery, i.e., trabeculectomy and tube shunt implants. The surgery can be either a single operation with multiple procedures performed at the same sitting or staged cataract and glaucoma surgery. Phacoemulsification alone reduces IOP, with the extent of IOP reduction varying between different studies, but is approximately $4 \mathrm{mmHg}(16.5 \%)$ based on the Ocular Hypertension Treatment Study [9]. This IOP-lowering effect is directly proportional to the IOP prior to surgery. If the patient has a component of angle closure, then cataract removal may be indicated even with 20/20 acuity.

\subsubsection{Target IOP}

In patients requiring a target IOP below $12 \mathrm{mmHg}$, Trabectome is not ideal. Devices that are designed to remove or bypass the trabecular meshwork by shunting fluid to Schlemm's canal theoretically cannot achieve IOP below the episcleral venous pressure, $8-10 \mathrm{mmHg}$ [10]. In patients requiring an IOP of $12 \mathrm{mmHg}$ or less, the procedures that may have a higher chance of success create a communication between the anterior chamber and subconjunctival space, e.g., subconjunctival MIGS devices or conventional glaucoma surgery (trabeculectomy, tube shunt device [e.g., Baerveldt or Ahmed glaucoma implant]).

In patients with a target IOP of approximately $15-17 \mathrm{mmHg}$, Trabectome surgery could be considered. Numerous studies have shown IOP decreases to the 15-17 mmHg range with both phaco-Trabectome and with Trabectome-alone [1114]. Mizoguchi et al. reported that the Trabectome failure rate was higher in the eyes with a preoperative IOP $<18 \mathrm{mmHg}$ and lower in those with a preoperative IOP of 18-22 mmHg [15]. Successful outcomes have been reported in studies with higher baseline IOP as well, such as $37.6 \pm 6.6 \mathrm{mmHg}$ in a study by Akil et al. [16] and $31.6 \pm 9.9 \mathrm{mmHg}$ by Shoji et al. [17]. These studies suggest that the higher the baseline IOP, the greater the percentage reduction in IOP.

\subsubsection{Trabectome Can Be Performed in Patients with Narrow Angles}

Trabectome has traditionally been reserved for patients with open angles (at least Shaffer grade 3) because it was thought that the failure rate would increase in patients with narrower angles and that the procedure would be difficult to perform safely. In Trabectome-alone cases and phaco-Trabectome combined cases, Bussel et al. found no significant difference in IOP reduction between eyes with Shaffer grade 3 or greater versus those with grade 2 or less. This would allow many patients who were previously not deemed to be MIGS candidates to be able to benefit from Trabectome [18]. 
In Chinese patients, Lee et al. reported that despite seeing an open-angle configuration on gonioscopy, the small dimensions of the anterior segment make it difficult to maneuver the Trabectome handpiece in the eye without damaging ocular structures such as the iris. Therefore, they only performed Trabectome surgery on pseudophakic Chinese eyes. Even in pseudophakic Chinese eyes, the view of the treated area of Schlemm's canal was obscured by normal iris (not peripheral anterior synechiae) in some eyes by 1 month after Trabectome surgery, as a consequence of the small anterior segment dimensions. However, this was not correlated with an IOP increase. [19].

\subsubsection{Contraindications}

Contraindications to trabectome surgery include neovascularization of the anterior chamber angle, glaucoma secondary to elevated episcleral venous pressure, chronic angle closure, and active uveitis [20]. Poor visualization of the trabecular meshwork or a very narrow anterior chamber angle increases the risk of damage to surrounding ocular structures.

\subsection{Results of Trabectome}

\subsubsection{Quality of Evidence}

While Trabectome surgery is widely performed, it is important to note the paucity of high-quality evidence. In 2016, Hu et al. conducted a Cochrane review of all published Trabectome studies. They included only randomized controlled trials resulting in the exclusion of all 113 published reports on Trabectome. A single randomized controlled trial was underway, NCT00901108, but has since been terminated [21]. The current compendium of Trabectome literature suffers from a lack of prospective, randomized, controlled studies. Other issues include industrysponsored studies with poor clinical trial procedures, e.g., enrollment, data collection and methods of patient selection. Variations in surgical techniques are likely to confound the surgical outcomes as each surgeon will ablate a different length of trabecular meshwork.

\subsubsection{Clinical Efficacy}

\subsubsection{Trabectome-Alone}

In 2005, the first clinical study by Minckler et al. reported results of Trabectomealone in 15 patients with primary and secondary open-angle glaucoma, who were recruited from a clinical practice in Tijuana, Mexico. At 1 year, the IOP decreased from $22.6 \pm 4.7 \mathrm{mmHg}$ to $16.3 \pm 2.0 \mathrm{mmHg}$, with a reduction in the mean number of medications from 1.2 to 0.1 [22]. 
Table 4.1 Commonly used Success and Failure criteria

Success criterion: postoperative IOP $<21 \mathrm{mmHg}$ and reduction of IOP $\geq 20 \%$ from baseline. Failure was determined if these criteria were not met on two consecutive visits after postoperative month 3

Failure: success criterion not met and/or need for additional glaucoma surgery

The largest data set comes from the global Trabectome study database sponsored by the device manufacturer, NeoMedix, which analyzes the first 20 cases of any Trabectome surgeon who voluntarily sends in de-identified clinical data as postmarket surveillance. This database is stored by the company, and the most recent update was published in 2014, which included 5435 cases of Trabectome-alone and phaco-Trabectome procedures, with up to 90 months of follow-up. On average, the IOP was reduced from $23.0 \pm 7.9 \mathrm{mmHg}$ to $16.5 \pm 3.8 \mathrm{mmHg}$ (29\% reduction) and the number of glaucoma medications was reduced from $2.6 \pm 1.3$ to $1.6 \pm 1.3(38 \%)$ [23]. However, a large number of patients were lost to follow-up with incomplete data from the first postoperative day, hence limiting the generalizability of these results.

In 2008, Minckler et al. published a follow-up study to update their results [13]. They adapted the success criteria from the Tube versus Trabeculectomy Study to evaluate Trabectome surgery (Table 4.1). This criteria has been adopted by most subsequent Trabectome studies. Although Minckler et al. noted the success rate of Trabectome after 12 months to be 50\%, the 1-year success rates have varied significantly in different studies, ranging from 36\% [24] to 94\% [25]. The study which reported a 1 -year success rate of $36 \%$ found that this decreased to $22.4 \%$ by 2 years [24]. In many of the aforementioned studies, the high dropout rates are likely to introduce selection bias, limiting the generalizability of the study results.

These studies use Kaplan-Meier survival plots to display the success rate over time. The significance of the plots is difficult to interpret because most of the Kaplan-Meier survival plots only show the success rate, but do not indicate the number of patients at each follow-up visit [23]. Inter-study results may vary as a consequence of different study designs, surgical technique, population differences between study groups, or different patient evaluation protocols (e.g., masked vs. unmasked IOP readings) [6-8, 11, 15-17, 22-24, 26].

\subsubsection{Trabectome-Alone Versus Trabeculectomy with Mitomycin C}

In a retrospective cohort study, Jea et al. compared Trabectome to trabeculectomy with mitomycin $\mathrm{C}$. This cohort study had a very low rate of participant dropout. After 24 months, IOP decreased from $28.1 \pm 8.6 \mathrm{mmHg}$ to $15.9 \pm 4.5 \mathrm{mmHg}$ in the Trabectome group versus $26.3 \pm 10.9 \mathrm{mmHg}$ to $10.2 \pm 4.1 \mathrm{mmHg}$ in the trabeculectomy group; $43.5 \%$ of Trabectome eyes and $10.8 \%$ of trabeculectomy eyes required subsequent glaucoma surgery. Two-year success rates were $22.4 \%$ and $76.1 \%$ in the Trabectome and trabeculectomy groups respectively, demonstrating a lower success rate of Trabectome compared to trabeculectomy. Younger age and lower preoperative IOP were risk factors for Trabectome failure [24]. The results were later corroborated by Sit et al. [27]. 


\subsubsection{Phaco-Trabectome Versus Trabectome-Alone}

Neiweem et al. published an algorithm to calculate anticipated IOP after Trabectome. They found that phacoemulsification plays a minimal role in IOP reduction when performed with Trabectome surgery. They calculated that adding phacoemulsification to Trabectome surgery lowers final IOP by only $0.73 \pm 0.32 \mathrm{mmHg}$ [28]. Similarly, studies have shown that there is no significant difference in the mean postoperative IOP and the mean number of hypotensive medications for phaco-Trabectome compared with Trabectome-alone [23, 25]. However, these findings are contradictory to the results of randomized control trials comparing cataract surgery alone to cataract surgery combined with other MIGS procedures, including iStent, CyPass, and Hydrus implantation.

Other studies have reported that the success rate of phaco-Trabectome is higher than that of Trabectome-alone. In a meta-analysis by Kapowitz et al., the mean 1 -year success rate of Trabectome-alone was $61 \pm 17 \%(n=5$ studies) and that of phaco-Trabectome was $85 \pm 17 \%$ ( $n=6$ studies) [29].

\subsubsection{Phaco-Trabectome Versus Phaco-iStent}

Three nonrandomized retrospective studies have compared phaco-Trabectome to phacoemulsification with two iStents [12-14]. In all three studies, there were no statistical differences between the postoperative IOP and the number of medications between the two groups. Lavia et al. came to the same conclusion in a meta-analysis [30].

\subsubsection{Preoperative Glaucoma Severity on Surgical Outcome}

It is thought that MIGS is most effective for mild-to-moderate glaucoma. Roy et al. looked at the 1-year postoperative outcomes of 498 patients undergoing phacoTrabectome, and stratified results based on a pre-operative glaucoma severity index which incorporated pre-operative IOP, the number of hypotensive medications and visual field status. Severity was designated on a scale of 1 through 4 with a higher number indicating more severe disease. Success rates after 1 year were noted to be $98,93,96$, and $88 \%$ in groups 1 to 4 respectively, with a $1.69 \pm 0.2 \mathrm{mmHg}$ larger IOP reduction with each increase in group number. This study suggests that phacoTrabectome surgery can be successful for severe glaucoma in carefully selected patients [31], though the efficacy of conventional glaucoma surgery (trabeculectomy and tube shunt implants) is certainly much more established in such patients.

\subsubsection{Other Potential Factors Affecting Efficacy}

\subsubsection{Does Prior Ineffective Selective Laser Trabeculoplasty (SLT) Alter Trabectome Outcomes?}

Vold et al. retrospectively compared the impact of Trabectome surgery on patients with and without prior SLT treatment [32]. No significant difference in IOP was found between the two groups. At 1 year, patients without prior SLT treatment 
$(n=177)$ had mean IOP of $16.5 \pm 4.0 \mathrm{mmHg}$, while those with prior SLT treatment $(n=58)$ had mean IOP of $15.7 \pm 3.0 \mathrm{mmHg}$.

Klamann et al. retrospectively compared the 6-month outcomes of eyes which underwent combined phaco-Trabectome 3 months after $360^{\circ}$ SLT, compared with eyes which did not undergo prior SLT. In eyes with POAG, there was no significant difference in IOP between the two groups at 6 months. In eyes with pigment dispersion and pseudoexfoliation, those that underwent prior SLT had a greater IOP reduction compared with those without previous SLT, though this difference was significant only at the 6-month time point and the number of hypotensive medications was similar between the two groups. In view of the small sample size, the lack of masking and a significant difference between the two groups being detectable only at a single time point, these results must be verified in a larger study before extrapolation to clinical practice [33].

\subsubsection{Trabectome for Patients with Secondary Open-Angle Glaucoma}

Pseudoexfoliation glaucoma, pigmentary glaucoma, and steroid-induced glaucoma have responded well to Trabectome surgery [26, 33-35]. Pahlitzsch et al. compared Trabectome-alone in POAG versus pseudoexfoliation glaucoma patients. They showed an IOP reduction from $19.10 \pm 4.11 \mathrm{mmHg}$ to $14.27 \pm 2.93 \mathrm{mmHg}$ and glaucoma medication reduction from $2.40 \pm 0.92$ to $1.77 \pm 1.00$ in the POAG group after 36 months. The pseudoexfoliation group had an IOP decrease from $22.49 \pm 9.40 \mathrm{mmHg}$ to $14.57 \pm 5.05 \mathrm{mmHg}$ and glaucoma medication reduction from $2.31 \pm 1.02$ to $1.75 \pm 0.91$ after 36 months [26]. Steroid-induced glaucoma has also responded well to Trabectome surgery $[34,35]$. Dang et al. showed that the success rates were $86 \%$ and $85 \%$ respectively for steroid-induced glaucoma and POAG at 1-year follow-up [34]. Trabectome surgery has been shown to be effective in uveitic glaucoma as well [36].

Some studies suggest that Trabectome surgery may be more successful in secondary open-angle glaucoma (SOAG) compared to POAG. In a Japanese study by Shoji et al., the success rate at 12 months for POAG patients $(n=80)$ and SOAG patients $(n=46)$ were $53.9 \pm 7.5 \%$ and $77.2 \pm 5.4 \%$, respectively [17]. In the SOAG group, 11 patients had steroid-induced glaucoma, 22 patients had pseudoexfoliation glaucoma, and 13 patients had uveitic glaucoma. In a study by Ting et al., success rates at 12 months for POAG and pseudoexfoliation glaucoma patients were 62.9 and $79.1 \%$ respectively $(P=0.004)$ [37]. Akil et al. found that Trabectome-alone success rates after 12 months were 86 and $92 \%$ for POAG and pigmentary glaucoma patients, respectively [16].

It is believed that Trabectome works well for secondary open-angle glaucoma, in particular pseudoexfoliation glaucoma and pigmentary glaucoma, because the site of greatest aqueous outflow resistance is at the trabecular meshwork (due to occlusion by pseudoexfoliative material or pigment), hence these eyes should respond well to trabecular meshwork removal. 


\subsubsection{Trabectome Can Be Performed in Patients with Previous Failed Trabeculectomy}

It was previously believed that following conventional glaucoma surgery, aqueous flow through the trabecular outflow pathway decreased significantly. Bussel et al. showed that Trabectome surgery can be beneficial with or without phacoemulsification in eyes with failed trabeculectomy [38]. At 1 year postoperatively, the mean IOP of the Trabectome-only group decreased by $28 \%$ from a baseline of $23.7 \mathrm{mmHg}$ while that of the phaco-Trabectome group decreased by $19 \%$ from a baseline of $20 \mathrm{mmHg}$. The mean number of hypotensive medications decreased from 2.8 to 2.0 in the Trabectome-only group and from 2.5 to 1.6 in the phaco-Trabectome group.

\subsubsection{Intraoperative Prognosticator for Postoperative Outcome}

Fellman et al. suggested that a prominent post-Trabectome episcleral venous fluid wave may be a reliable biomarker for successful Trabectome surgery [39]. Immediately after the Trabectome procedure, the extent of episcleral venous blanching adjacent to the Trabectome site was noted in response to increased IOP, achieved by adjusting the irrigation level within the anterior chamber. Preoperatively, patients had mean IOP of $19.3 \pm 5.1 \mathrm{mmHg}$ on $2.7 \pm 0.9$ medications. Patients with a prominent fluid wave had a post-Trabectome mean IOP of $13.3 \pm 2.7 \mathrm{mmHg}$ on $1.4 \pm 1.2$ medications while patients with a poor fluid wave had a post-Trabectome mean IOP of $18.4 \pm 3.1 \mathrm{mmHg}$ on $2.9 \pm 0.9$ medications at 12 months follow-up. However, the study did not report the preoperative IOP and number of medications separately for the two cohorts, hence it was possible that the difference in postoperative outcomes between the groups might be partly attributable to preoperative differences. A reliable method of assessing the preoperative episcleral venous flow has not been established and the magnitude of increase in episcleral venous flow secondary to Trabectome surgery is unclear. Furthermore, the value of this sign for preoperative prognostication is limited as it can only be observed after the procedure has been performed.

\subsection{Complications of Trabectome}

In 2008, Minckler et al. reported the rate of complications associated with Trabectome surgery from a review of 1127 patients [40]. In this study, 17 patients $(1.5 \%)$ had hypotony (IOP $<5 \mathrm{mmHg}$ ) at day 1,65 patients $(5.8 \%)$ had an IOP spike (IOP $>10 \mathrm{mmHh}$ above baseline), 874 patients (77.6\%) had intraoperative blood reflux, and one patient had aqueous misdirection $(0.09 \%)$. Infection, wound leak, bleb formation, choroidal effusion, choroidal hemorrhage, or visual acuity decrease of more than two lines did not occur in any of the patients [40] (Table 4.2).

In the study by Francis et al. which included 304 eyes that underwent phacoTrabectome, an IOP spike of $10 \mathrm{mmHg}$ or greater occurred in 26 eyes (8.6\%) at day 1 and in 6 eyes (2.0\%) at week 1 after the surgery. Francis et al. also noted minor iris injury from the Trabectome tip in four cases (1.3\%) [42].

Mizoguchi et al. reported that the complications after Trabectome surgery included microhyphema (76.8\%), hyphema (23.2\%), IOP spike >10 mmHg (4.9\%), decrease in VA greater than two lines (1.2\%), and cataract progression (1.2\%) [15]. 
Table 4.2 Complications of Trabectome surgery
Intraoperative blood reflux (78\% of 1127 cases) [40]; 92\% of 557 [41]

Postoperative hyphema (23.2\% of 82 cases) [15]

Peripheral anterior synechiae (14.0\% of 101 cases) [6]

Transient IOP spike (5.8\% of 1127 cases) [40]

IOP at 1 week $>10 \mathrm{mmHg}$ higher than preop (2.0\% of 304 cases) [42]

Hypotony (1.5\% of 1127 cases) [40]

Cyclodialysis cleft, low incidence rate [43, 44]

Iris injury (1.3\% of 304 cases) [42]

Cataract progression (1.2\% of 82 cases) [15]

Serious complications are rare after Trabectome surgery, with the incidence similar to that associated with cataract surgery [29]. Maeda et al. showed that there was no statistically significant difference in corneal endothelial cell count before and after Trabectome surgery [45].

The most common reasons for surgical failure are incomplete or improper removal of the trabecular meshwork, ablation of the wrong site hence damaging the ciliary body, and damage to Schlemm's canal or surrounding tissue which results in scarring. While blood reflux from the collector channels is common, surgical intervention for hyphema is rare [46]. Peripheral anterior synechiae have been observed in nearly a quarter of patients in some studies [6].

Berk et al. documented the first case of cyclodialysis cleft secondary to a complicated Trabectome procedure which resulted in hypotony requiring direct suture cyclopexy [43]. While there have been a few reports on cyclodialysis cleft formation after Trabectome surgery, it may be an underreported complication. A cyclodialysis cleft may be difficult to identify in the clinic postoperatively, as the associated hypotony may narrow the anterior chamber angle and hinder visualization of the ciliary body band. Ultrasound biomicroscopy may be helpful in identifying cyclodialysis clefts postoperatively [44]. The cleft is most easily visualized immediately after creation when the eye is still pressurized from the Trabectome infusion.

One should suspect a cleft intraoperatively when there is excessive bleeding or when the postoperative IOP reduction is more than expected. Closure of the cleft typically results in a transient IOP spike which usually lasts about 4-5 days.

\subsection{After Trabectome Has Failed}

\subsubsection{Does Trabectome Surgery Impact Future Glaucoma Surgery Outcomes?}

Trabectome surgery spares the conjunctiva and Jea et al. showed that failed Trabectome surgery did not impact the success of subsequent trabeculectomy [47]. However, after failed Trabectome surgery, subsequent SLT has limited benefit [48]. 


\subsubsection{Gonioscopy-Assisted Transluminal Trabeculotomy (GATT) Procedure as a Potential Treatment Option After Failed Trabectome Surgery}

GATT is adapted from ab-externo trabeculotomy to create a safer and easier approach to access Schlemm's canal. A microcatheter is fed into Schlemm's canal more than $360^{\circ}$ using an ab-interno approach through a trabecular incision. GATT has been shown to be effective at decreasing medication reliance and improving IOP in both primary and secondary glaucoma $[49,50]$. Hyphema is the most frequent complication and is present in $34 \%$ of eyes 1 week after the surgery.

In patients who initially responded to Trabectome surgery but subsequently developed elevated IOP again, GATT may be beneficial as it allows aqueous to access a larger segment of Schlemm's canal than Trabectome surgery. The GATT fiber optic probe may be inserted into the Schlemm's canal through the previous Trabectome trabecular incision.

\subsection{Conclusion}

Trabectome surgery bypasses the trabecular meshwork and allows aqueous to flow directly from the anterior chamber to the Schlemm's canal. This is a bleb-less procedure which spares the conjunctiva. Multiple studies have shown that Trabectome surgery results in a reduction in IOP and the number of ocular hypotensive agents, though the efficacy is significantly less than conventional glaucoma surgery. Trabectome surgery has a favorable safety profile compared with conventional filtration surgery, with the most common complication being intraoperative and postoperative bleeding. Disadvantages of Trabectome surgery include the modest efficacy, which is limited by episcleral venous pressure, and the difficulty in targeting treatment to the segment of Schlemm's canal with the highest concentration of functional collector channels. Additional research is required to understand how the efficacy of Trabectome surgery can be maximized.

\section{References}

1. Brubaker RF. Targeting outflow facility in glaucoma management. Surv Ophthalmol. 2003;48(Suppl 1):S17-20.

2. Lutjen-Drecoll E. Functional morphology of the trabecular meshwork in primate eyes. Prog Retin Eye Res. 1999;18(1):91-119.

3. Chihara E, Nishida A, Kodo M, Yoshimura N, Matsumura M, Yamamoto M, et al. Trabeculotomy ab externo: an alternative treatment in adult patients with primary open-angle glaucoma. Ophthalmic Surg. 1993;24(11):735-9.

4. Rosenquist R, Epstein D, Melamed S, Johnson M, Grant WM. Outflow resistance of enucleated human eyes at two different perfusion pressures and different extents of trabeculotomy. Curr Eye Res. 1989;8(12):1233-40.

5. Seibold LK, Soohoo JR, Ammar DA, Kahook MY. Preclinical investigation of ab interno trabeculectomy using a novel dual-blade device. Am J Ophthalmol. 2013;155(3):524-9.e2. 
6. Minckler D, Baerveldt G, Ramirez MA, Mosaed S, Wilson R, Shaarawy T, et al. Clinical results with the Trabectome, a novel surgical device for treatment of open-angle glaucoma. Trans Am Ophthalmol Soc. 2006;104:40-50. Pubmed Central PMCID: 1809927

7. Francis BA, See RF, Rao NA, Minckler DS, Baerveldt G. Ab interno trabeculectomy: development of a novel device (Trabectome) and surgery for open-angle glaucoma. J Glaucoma. 2006;15(1):68-73.

8. Mosaed S, Dustin L, Minckler DS. Comparative outcomes between newer and older surgeries for glaucoma. Trans Am Ophthalmol Soc. 2009;107:127-33. Pubmed Central PMCID: 2814584

9. Mansberger SL, Gordon MO, Jampel H, Bhorade A, Brandt JD, Wilson B, et al. Reduction in intraocular pressure after cataract extraction: the Ocular Hypertension Treatment Study. Ophthalmology. 2012;119(9):1826-31. Pubmed Central PMCID: 3426647

10. Ophthalmology AAo. Elevated Episcleral Venous Pressure. 2017. https://www.aao.org/bcscsnippetdetail.aspx ?id=52ea963b-4164-480a-880b-6a79ed3f4772.

11. Akil H, Chopra V, Huang AS, Swamy R, Francis BA. Short-term clinical results of ab interno trabeculotomy using the Trabectome with or without cataract surgery for open-angle glaucoma patients of high intraocular pressure. J Ophthalmol. 2017;2017:8248710. Pubmed Central PMCID: 5412169

12. Gonnermann J, Bertelmann E, Pahlitzsch M, Maier-Wenzel AB, Torun N, Klamann MK. Contralateral eye comparison study in MICS \& MIGS: Trabectome(R) vs. iStent inject(R). Graefes Arch Clin Exp Ophthalmol. 2017;255(2):359-65.

13. Khan M, Saheb H, Neelakantan A, Fellman R, Vest Z, Harasymowycz P, et al. Efficacy and safety of combined cataract surgery with 2 trabecular microbypass stents versus ab interno trabeculotomy. J Cataract Refract Surg. 2015;41(8):1716-24.

14. Kurji K, Rudnisky CJ, Rayat JS, Arora S, Sandhu S, Damji KF, et al. Phaco-trabectome versus phaco-iStent in patients with open-angle glaucoma. Canad J Ophthalmol J. 2017;52(1): 99-106.

15. Mizoguchi T, Nishigaki S, Sato T, Wakiyama H, Ogino N. Clinical results of Trabectome surgery for open-angle glaucoma. Clin Ophthalmol. 2015;9:1889-94. Pubmed Central PMCID: 4607056

16. Akil H, Chopra V, Huang A, Loewen N, Noguchi J, Francis BA. Clinical results of ab interno trabeculotomy using the Trabectome in patients with pigmentary glaucoma compared to primary open angle glaucoma. Clin Exp Ophthalmol. 2016;44(7):563-9.

17. Shoji N, Kasahara M, Iijima A, Takahashi M, Tatsui S, Matsumura K, et al. Short-term evaluation of Trabectome surgery performed on Japanese patients with open-angle glaucoma. Jpn J Ophthalmol. 2016;60(3):156-65.

18. Bussel II, Kaplowitz K, Schuman JS, Loewen NA, Trabectome SG. Outcomes of ab interno trabeculectomy with the trabectome by degree of angle opening. $\mathrm{Br} \mathrm{J}$ Ophthalmol. 2015;99(7):914-9. Pubmed Central PMCID: 4501175

19. Lee JW, Yick DW, Tsang S, Yuen CY, Lai JS. Efficacy and safety of Trabectome surgery in Chinese open-angle glaucoma. Medicine. 2016;95(15):e3212. Pubmed Central PMCID: 4839803

20. Hardik A, Parikh PR, Dhaliwal A, Kaplowitz KB, Loewen NA. Trabectome patient selection, preparation, technique, management, and outcomes. touch Ophthalmol. 2015;8(2):5. Epub 2015

21. Hu K, Gazzard G, Bunce C, Wormald R. Ab interno trabecular bypass surgery with Trabectome for open angle glaucoma. Cochrane Database Syst Rev. 2016;15(8):CD011693.

22. Minckler DS, Baerveldt G, Alfaro MR, Francis BA. Clinical results with the Trabectome for treatment of open-angle glaucoma. Ophthalmology. 2005;112(6):962-7.

23. Mosaed S. The first decade of global trabectome outcomes. touch Ophthalmol. 2014;8(2):113-9.

24. Jea SY, Francis BA, Vakili G, Filippopoulos T, Rhee DJ. Ab interno trabeculectomy versus trabeculectomy for open-angle glaucoma. Ophthalmology. 2012;119(1):36-42.

25. Okeke CO, Miller-Ellis E, Rojas M, Trabectome Study G. Trabectome success factors. Medicine. 2017;96(24):e7061. Pubmed Central PMCID: 5478308 
26. Pahlitzsch M, Davids AM, Zorn M, Torun N, Winterhalter S, Maier AB, et al. Three-year results of ab interno trabeculectomy (Trabectome): Berlin study group. Graefes Arch Clin Exp Ophthalmol. 2018;256:611-9.

27. Ahuja Y, Pyi SMK, Malihi M, Hodge DO, Sit AJ. Clinical results of ab interno trabeculotomy using the trabectome for open-angle glaucoma: the Mayo clinic series in Rochester, Minnesota. Am J Ophthalmol. 2013;156:97.

28. Neiweem AE, Bussel II, Schuman JS, Brown EN, Loewen NA. Glaucoma surgery calculator: limited additive effect of phacoemulsification on intraocular pressure in ab interno trabeculectomy. PLoS One. 2016;11(4):e0153585. Pubmed Central PMCID: 4831696

29. Kaplowitz K, Bussel II, Honkanen R, Schuman JS, Loewen NA. Review and meta-analysis of ab-interno trabeculectomy outcomes. Br J Ophthalmol. 2016;100(5):594-600.

30. Lavia C, Dallorto L, Maule M, Ceccarelli M, Fea AM. Minimally-invasive glaucoma surgeries (MIGS) for open angle glaucoma: a systematic review and meta-analysis. PLoS One. 2017;12(8):e0183142. Pubmed Central PMCID: 5574616

31. Roy P, Loewen RT, Dang Y, Parikh HA, Bussel II, Loewen NA. Stratification of phacotrabectome surgery results using a glaucoma severity index in a retrospective analysis. BMC Ophthalmol. 2017;17(1):30. Pubmed Central PMCID: 5360039

32. Vold SD, Dustin L, Trabectome Study G. Impact of laser trabeculoplasty on Trabectome(R) outcomes. Ophthalmic Surg Lasers Imaging. 2010;41(4):443-51.

33. Klamann MK, Gonnermann J, Maier AK, Bertelmann E, Joussen AM, Torun N. Influence of Selective Laser Trabeculoplasty (SLT) on combined clear cornea phacoemulsification and Trabectome outcomes. Graefes Arch Clin Exp Ophthalmol. 2014;252(4):627-31.

34. Dang Y, Kaplowitz K, Parikh HA, Roy P, Loewen RT, Francis BA, et al. Steroid-induced glaucoma treated with trabecular ablation in a matched comparison with primary open-angle glaucoma. Clin Exp Ophthalmol. 2016;44(9):783-8.

35. Ngai P, Kim G, Chak G, Lin K, Maeda M, Mosaed S. Outcome of primary trabeculotomy ab interno (Trabectome) surgery in patients with steroid-induced glaucoma. Medicine. 2016;95(50):e5383. Pubmed Central PMCID: 5268022

36. Anton A, Heinzelmann S, Ness T, Lubke J, Neuburger M, Jordan JF, et al. Trabeculectomy ab interno with the Trabectome $(\mathrm{R})$ as a therapeutic option for uveitic secondary glaucoma. Graefes Arch Clin Exp Ophthalmol. 2015;253(11):1973-8.

37. Ting JL, Damji KF, Stiles MC, Trabectome Study G. Ab interno trabeculectomy: outcomes in exfoliation versus primary open-angle glaucoma. J Cataract Refract Surg. 2012;38(2):315-23.

38. Bussel II, Kaplowitz K, Schuman JS, Loewen NA, Trabectome Study G. Outcomes of ab interno trabeculectomy with the trabectome after failed trabeculectomy. Br J Ophthalmol. 2015;99(2):258-62. Pubmed Central PMCID: 4316927

39. Fellman RL, Feuer WJ, Grover DS. Episcleral venous fluid wave correlates with Trabectome outcomes: intraoperative evaluation of the trabecular outflow pathway. Ophthalmology. 2015;122(12):2385-91. e1

40. Minckler D, Mosaed S, Dustin L, Ms BF, Trabectome Study G. Trabectome (trabeculectomyinternal approach): additional experience and extended follow-up. Trans Am Ophthalmol Soc. 2008;106:149-59. discussion 59-60. Pubmed Central PMCID: 2646453

41. Jordan JF, Wecker T, van Oterendorp C, Anton A, Reinhard T, Boehringer D, et al. Trabectome surgery for primary and secondary open angle glaucomas. Graefes Arch Clin Ex Ophthalmol. 2013;251(12):2753-60. Pubmed Central PMCID: 3889259

42. Francis BA, Minckler D, Dustin L, Kawji S, Yeh J, Sit A, et al. Combined cataract extraction and trabeculotomy by the internal approach for coexisting cataract and open-angle glaucoma: initial results. J Cataract Refract Surg. 2008 Jul;34(7):1096-103.

43. Berk TA, An JA, Ahmed IIK. Inadvertent cyclodialysis cleft and hypotony following abinterno trabeculotomy using the Trabectome device requiring surgical repair. J Glaucoma. 2017;26(8):742-6.

44. Osman EA, AlMobarak F. Ciliochoroidal effusion with persistent hypotony after trabectome surgery. Indian J Ophthalmol. 2015;63(3):272-4. Pubmed Central PMCID: 4448246 
45. Maeda M, Watanabe M, Ichikawa K. Evaluation of trabectome in open-angle glaucoma. J Glaucoma. 2013;22(3):205-8.

46. Kaplowitz K, Schuman JS, Loewen NA. Techniques and outcomes of minimally invasive trabecular ablation and bypass surgery. Br J Ophthalmol. 2014;98(5):579-85. Pubmed Central PMCID: 4108346

47. Jea SY, Mosaed S, Vold SD, Rhee DJ. Effect of a failed trabectome on subsequent trabeculectomy. J Glaucoma. 2012;21(2):71-5.

48. Töteberg-Harms M, Rhee DJ. Limited success of selective laser trabeculoplasty following failed combined phacoemulsification cataract extraction and $a b$ interno trabeculectomy (Trabectome). Am J Ophthalmol. 2013;156:936-40. Aug 7 Epub ahead of print

49. Grover DS, Godfrey DG, Smith O, Shi W, Feuer WJ, Fellman RL. Outcomes of Gonioscopyassisted Transluminal Trabeculotomy (GATT) in eyes with prior incisional glaucoma surgery. J Glaucoma. 2017;26(1):41-5.

50. Grover DS, Godfrey DG, Smith O, Feuer WJ, Montes de Oca I, Fellman RL. Gonioscopyassisted transluminal trabeculotomy, ab interno trabeculotomy: technique report and preliminary results. Ophthalmology. 2014;121(4):855-61.

Open Access This chapter is licensed under the terms of the Creative Commons Attribution 4.0 International License (http://creativecommons.org/licenses/by/4.0/), which permits use, sharing, adaptation, distribution and reproduction in any medium or format, as long as you give appropriate credit to the original author(s) and the source, provide a link to the Creative Commons license and indicate if changes were made.

The images or other third party material in this chapter are included in the chapter's Creative Commons license, unless indicated otherwise in a credit line to the material. If material is not included in the chapter's Creative Commons license and your intended use is not permitted by statutory regulation or exceeds the permitted use, you will need to obtain permission directly from the copyright holder.

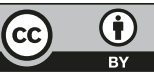

